\title{
Extra-adrenal glucocorticoid synthesis at epithelial barriers
}

\author{
Truong San Phan ${ }^{1} \cdot$ Verena M. Merk ${ }^{1} \cdot$ Thomas Brunner $^{1}$
}

\begin{abstract}
Epithelial barriers play an important role in the exchange of nutrients, gases, and other signals between our body and the outside world. However, they protect it also from invasion by potential pathogens. Defective epithelial barriers and associated overshooting immune responses are the basis of many different inflammatory disorders of the skin, the lung, and the intestinal mucosa. The anti-inflammatory activity of glucocorticoids has been efficiently used for the treatment of these diseases. Interestingly, epithelia in these tissues are also a rich source of endogenous glucocorticoids, suggesting that local glucocorticoid synthesis is part of a tissue-specific regulatory circuit. In this review, we summarize current knowledge about the extra-adrenal glucocorticoid synthesis at the epithelial barriers of the intestine, lung and the skin, and discuss their relevance in the pathogenesis of inflammatory diseases and as therapeutic targets.
\end{abstract}

Several organs from endodermal and ectodermal origins are geared with an epithelial layer providing a physical separation and protection of the organ from the environment. Among them, different types of epithelia exist, such as the mucosal epithelium in the gastrointestinal tract, the respiratory epithelium of the lung or the keratinized stratified squamous epithelium in the skin. The mucosal epithelia in the lung and in the intestine consist of a single layer of epithelial cells, whereas the cutaneous epithelium is structured and stratified in multiple layers with a superficial cornified stratum. The epithelial barriers of these organs are not only differentially structured, but also serve different functions. While the physiological function of the intestinal and pulmonary epithelium is the uptake and exchange of nutrients and gases, respectively, the skin plays a crucial role in protecting the inner parts of the body from the environment, next to communication of the body with its environment via sensory mechanisms. Although all three epithelial surfaces fulfill different functions, at the front line between our body and the outside world they are all exposed to potential pathogen invasion. Thus, all three epithelia also contain a complex immune system with specialized immune cell subsets in order to cope with pathogen

$\triangle$ Thomas Brunner

thomas.brunner@uni-konstanz.de

1 Biochemical Pharmacology, Department of Biology, University of Konstanz, Konstanz, Germany invasion and associated inflammatory stress. In this respect, all three organs are subject to a broad range of inflammatory disorders. Specifically, combination of epithelial barrier disruption in these organs and uncontrolled immune responses against luminal or superficial microbiota may result in inflammatory disorders, not only causing local tissue-restricted damage, but affecting also other organs and possibly the entire homeostasis of the body. Pharmacological treatments of different inflammatory disorders of the intestine, lung, and skin include often anti-inflammatory agents and steroid-based applications to restore tissue and epithelial barrier homeostasis. Steroids are, however, not only active ingredients of anti-inflammatory drugs, but endogenous hormones produced by various tissues. In this review, we therefore discuss the importance of endogenous and locally produced steroids in the lung, the intestine and the skin, and most importantly their role in controlling homeostatic processes at these epithelial barriers.

\section{Glucocorticoids}

In 1950, P.S. Hench, E. Kendall, and T. Reichstein were awarded for the Nobel Prize in Physiology for the discovery and characterization of the so-called adrenal cortical hormones, which are known to this day as glucocorticoids (GC). Since more than 50 years, GC have been extensively used to treat a large variety of inflammatory and autoimmune diseases, but clinical applications of GC also included organ transplantation as well as the therapy of 
tumors, especially leukemias. The relevance of the immunosuppressive effect of GC mediated by the glucocorticoid receptor (GR) is to this day undeniable, which led to intense search for the underlying mechanism and the relevance of transcriptional GR targets. The GR is a member of the nuclear receptor superfamily 3 (NR3). It mainly exerts its action through transcriptional activation or repression of GC-responsive genes by directly binding to positive or negative GC response elements (GRE) in the enhancer or promoter region of target genes. Apart from the direct control of target genes, the GR mediates its antiinflammatory activity also by binding to and thereby neutralizing transcription factors involved in the regulation of inflammation, such as AP-1 or NFkB [1-4]. Next to the direct or indirect control of gene products involved in inflammatory processes, such as tumor necrosis factor (TNF), IL-1 $\beta$, and other pro-inflammatory cytokines, GC may regulate inflammation also by inducing apoptosis in immune cells, and thereby reducing their number and action in inflammatory diseases. In this respect, GR-induced expression of the pro-apoptotic Bcl-2 homolog Bim (Bcl2L11) and/or GR-mediated inhibition of NFkB-regulated pro-survival genes appear to be critical underlying mechanisms [5, 6]. Since the discovery of the adrenal GC, several synthetic and highly potent GC for the treatment of inflammatory diseases have been developed. Compared to the synthetic GC, endogenous GC are known to have rather broad effects, but to be less potent than synthetic homologs. De novo synthesis of systemic GC is known to be locally restricted to the adrenal cortex. Briefly, in the first step cholesterol is transported to the inner mitochondrial membrane where its side chain is cleaved by CYP11A1 to produce pregnenolone, the first precursor of all steroids (Fig. 1). After dehydroxylation by $3 \beta$-HSD, further steps involve hydroxylation by CYP21A2 and CYP11B1, encoding the final enzyme $11 \beta$-hydroxylase in the de novo GC synthesis (Fig. 1). Furthermore, the expression of CYP17A1 and subsequent hydroxylation at C17 of pregnenolone and progesterone in human enables the exclusive synthesis of the potent GC cortisol (Fig. 1). In contrast, activation and deactivation of circulating GC metabolites occur through the $11 \beta$-hydroxysteroid dehydrogenase

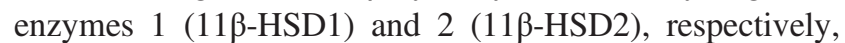
which are expressed in various tissue and cell types, providing and restricting local amounts of $\mathrm{GC}$ at the same time (Fig. 1). In addition to the adrenal cortex, studies of the past 2 decades revealed that several extra-adrenal tissues are capable of autonomously producing bioactive GC. Whereas some tissues preferentially synthesize GC de novo, others mainly reactivate GC metabolites through an $11 \beta$-HSD1dependent process. In the following chapters, we provide an update in GC synthesis at local epithelial barriers in comparison to the adrenal GC synthesis.

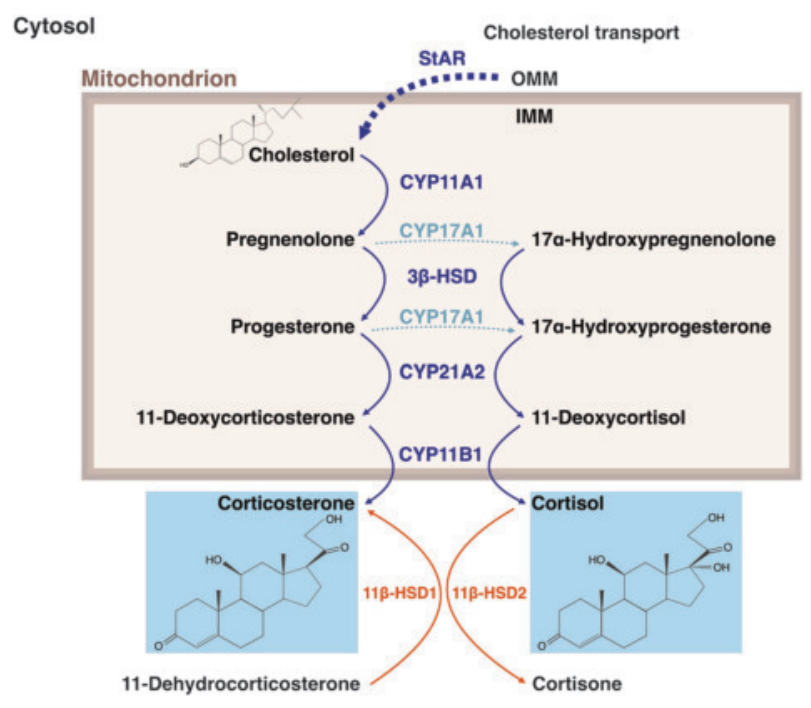

Fig. 1 The glucocorticoid synthesis pathway. In the de novo glucocorticoid synthesis pathway, the cholesterol transport from the outer mitochondrial membrane (OMM) to the inner mitochondrial membrane (IMM) represents the rate-limiting process and occurs via the steroidogenic acute regulatory protein (StAR). Cholesterol is then cleaved by the cholesterol side-chain cleavage enzyme (CYP11A1) to pregnenolone. After dehydroxylation by the $3 \beta$-hydroxysteroid dehydrogenase ( $3 \beta$-HSD), progesterone is hydroxylated by the steroid 21 hydroxylase (CYP21A2). With the expression of the steroid 17ahydroxylase (CYP17A1), human steroidogenic cells are able to produce $17 \alpha$-hydroxypregnenolone and $17 \alpha$-hydroxyprogesterone via the hydroxylation of pregnenolone and progesterone, respectively. In the final step, 11-deoxycorticosterone and/or 11-deoxycortisol are hydroxylated by the $11 \beta$-hydroxylase (CYP11B1) creating thereby bioactive glucocorticoids (GC). Bioactive GC, corticosterone or cortisol, can be inactivated by the $11 \beta$-hydroxysteroid dehydrogenase 2 (11 $\beta$-HSD2) in the cytosol to 11-dehydrocorticosterone or cortisone, respectively. Vice versa, inactive GC metabolites can also be reactivated by $11 \beta$ hydroxysteroid dehydrogenase 1 (11 $\beta$-HSD1) in the cytosol

\section{Adrenal glucocorticoid synthesis}

Endogenous GC are mainly produced in the adrenal glands via a series of enzymatic steps from cholesterol. Adrenal GC are released into the circulation, where they reach distant tissues and thus act systemically. Production and secretion of adrenal GC is under the control of the central neuroendocrine system, which is also known as the hypothalamic-pituitary-adrenal (HPA) axis, representing a global stress response system. Rapid production of adrenalderived GC upon inflammatory, psychological and physical stress reactions, or through circadian rhythm-mediated regulations, begins with the secretion of corticotropin releasing hormone $(\mathrm{CRH})$ into the hypophysial portal vessels by the paraventricular nucleus (PVN) in the hypothalamus [7]. Through the hypophysial portal vessels, CRH reaches the anterior pituitary gland and binds to the Gprotein-coupled receptor $\mathrm{CRH}$ receptor 1 (CRH-R1) stimulating thereby the production and release of the adrenocorticotropic hormone $(\mathrm{ACTH})$, which derives from the 
precursor protein proopiomelanocortin (POMC) [7]. Classically, ACTH is secreted into the circulation and binds to the melanocortin 2 receptor (MC2R) in the Zona fasciculata of the adrenal cortex, where it promotes the synthesis of GC, such as cortisol (humans) and corticosterone (rodents). In addition to the stress-induced adrenal GC response, systemic GC synthesis lies under the control of the circadian rhythm through the biological clock, which is centrally directed by the suprachiasmatic nucleus (SCN) in the hypothalamus $[8,9]$. As extensively reviewed by Kalsbeek et al., light/dark or day/night cycles, respectively, dictate the circadian control of adrenal GC secretion via two different pathways: (1) the SCN-PVN-HPA axis pathway mediated by $\mathrm{CRH}$ and $\mathrm{ACTH}$, and (2) the direct neural pathway via the autonomic nervous system [10]. However, it was described that circadian rhythm-controlled adrenal GC secretion seems to rely on the SCN via the direct autonomic nervous system, whereas the SCN-PVN-HPA axis pathway seems to be more relevant for stress-induced GC responses [10]. Importantly, the adrenal gland is also considered as a peripheral clock, which is able to orchestrate other peripheral clocks through humoral regulation via GC and mineralocorticoid (MC) secretion. It thereby represents an extended arm of the central clock in the SCN and contributes to synchronization of peripheral clocks by providing them a time cue. The role of circadian rhythmcontrolled adrenal GC and MC seems to be broader compared to the stress response system, as it daily prepares the system and its organs for enhanced energy supplementation to increase the body activity in a time-dependent and light/ dark-dependent manner, respectively [10].

Compared to the circadian rhythm-controlled GC secretion, the stress response system is regulated via the HPA axis, specifically by the steering hormone ACTH, which is able to rapidly provide adrenal GC through fast transcriptional and (post)translational control of the steroidogenic acute regulatory protein (StAR) $[11,12]$. Specifically, it was shown that in the acute response phase ACTH-mediated activation of the cAMP/protein kinase A (PKA) signaling pathway results in increased StAR activity, which initiates thereby the de novo GC synthesis by transporting cholesterol from the outer to the inner mitochondrial membrane, representing the rate-limiting step in steroid synthesis [13] (Fig. 1). Furthermore, a detailed review provided by Ruggiero and Lalli [14] also describes that ACTH critically drives the transcription of steroidogenic enzymes through different nuclear factors, including basal and inducible steroidogenic regulators. Worth mentioning is the steroidogenic factor 1 (SF-1 and NR5A1), an orphan nuclear receptor from the nuclear receptor 5 superfamily (NR5), which is critically involved in the development and differentiation of the adrenal glands and the gonads, but also in the steroidogenesis of these organs through transcriptional regulation of steroidogenic genes, in both, the HPA and the HPG axis [15-17]. SF-1 transcriptionally controls the expression of several cytochrome $\mathrm{P} 450$ enzymes by binding to either SF-1-binding sequences or cAMP response elements (CRE) in their promoter region [14]. By interacting with co-repressors or co-activators, $\mathrm{SF}-1$ regulates the basal expression of StAR, CYP11A1, CYP17A1, CYP21, HSD3B2, CYP11B2, and CYP11B1, but also promotes their ACTH/cAMP-induced expression and associated adrenal steroidogenesis [14]. Next to SF-1, there are numerous other nuclear factors, such as the CRE binding protein (CREB), CRE modulator, or activating transcription factor, which recognize the CRE sites and mediate cAMP/ PKA-regulated steroidogenesis. As highlighted by Ruggiero and Lalli [14], although CREB was reported to be the main transcription factor mediating cAMP/PKA-dependent steroidogenic enzyme expression, other CRE nuclear factors were reported to compensate each other in the absence of another. However, for certain steroidogenic gene transcripts different CREB members seem to be more relevant, such as Cypl1b1 in mice or CYP11B1 in humans [14, 18, 19].

\section{Extra-adrenal glucocorticoid synthesis}

Research of the last two decades revealed a number of other organs that express all steroidogenic enzymes necessary for de novo GC synthesis from cholesterol or GC reactivation from inactive metabolites [20]. Various tissues, including the thymus, the intestine, the lung and the skin, are able to produce bioactive GCs, and there is also evidence for GC production in the brain and the vascular system [20]. In comparison to the adrenal glands, the overall amount of locally produced GCs in extra-adrenal tissues may be rather low, but the conclusion their role in regulation tissuespecific processes is irrelevant may be deceptive since locally produced GCs may accumulate at specific loci in extra-adrenal tissues. Although many extra-adrenal tissues are shown to express all the enzymes involved in de novo GC synthesis, both pathways, GC reactivation and de novo synthesis, seem to be differentially regulated in these steroidogenic tissues. While the skin and the intestine predominantly synthesize GC de novo, the lung was shown to preferentially reactivate corticosterone from dehydrocorticosterone via an 11 $\beta$-HSD1-catalyzed process [21]. In contrast, a recent study revealed that GC synthesis in thymic epithelial cells, which plays an important role in thymocyte development, is dependent on the $11 \beta$-hydroxylase-regulated de novo synthesis pathway [22].

Despite many remaining open questions regarding the (patho)physiological role of extra-adrenal GC synthesis, research from the last couple of years documented the importance of epithelial barrier-derived GC in the regulation 
of local immune responses and tissue homeostasis. We, therefore, highlight and review here the extra-adrenal GC synthesis in the intestine, the lung and the skin, and discuss novel concepts based on recent research in this field.

\section{Intestine}

The intestine is an endoderm-derived organ representing one of the largest epithelial surfaces of the body. It represents a functional barrier within the body separating the internal from the external environment. The intestinal mucosal barrier, which is formed by simple columnar epithelial cells, is constantly challenged by luminal substances and prevents the entry of microbes into the body. Geared with one of the fastest cell renewals in the body the epithelium simultaneously provides barrier function and anti-microbial defense to protect from invading pathogens while maintaining digestive and absorptive functions. The intestinal lumen is home of a plethora of diverse microbes, ranging from commensals to pathogens. The intestinal epithelium is protected by the mucus layer and anti-microbial substances. Furthermore, it is equipped with the largest immune system in our body. Peyer's patches and the mesenteric lymph nodes are lymphoid tissues harboring large numbers of protective and tolerance-inducing immune cells, whereas other resident immune cells are distributed within the epithelium (intraepithelial lymphocytes). As part of the digestive tract, a well-characterized function of the intestine is the uptake of nutrients, salts and water, and the secretion of mucus and digestive enzymes into the lumen. More recently, the intestine has also been recognized as an endocrine tissue [20, 23]. The intestinal epithelium expresses all steroidogenic enzymes required for de novo synthesis of GC and secretes functional immunoregulatory GC in response to immunological stress [23]. Although basal levels of intestinal GC were shown to be rather moderate, in vivo immune cell activation upon anti-CD3 antibody or lipopolysaccharides (LPS) injection results in a drastic increase in local GC synthesis (Fig. 2). These experiments revealed that activation of both, the adaptive as well as the innate immune system is able to trigger intestinal GC synthesis [23, 24]. While TNF $\alpha$ is a pro-inflammatory cytokine with important functions in the pathogenesis of intestinal inflammatory disorders, such as Crohn's disease and ulcerative colitis (UC), it seems to be also an important trigger of intestinal GC synthesis in response to T cell and macrophage activation [24, 25].

\section{Regulation of intestinal glucocorticoid synthesis}

Intestinal steroidogenesis was shown to be differentially regulated compared to other steroidogenic tissues, such as the adrenal glands or the lung. Most pronounced differences are found in the transcriptional regulation of tissue-specific steroidogenesis, and the signals triggering these processes. For example, while ACTH-induced intracellular increase in cAMP and associated activation of PKA is an important trigger of adrenal GC synthesis, intracellular increase of cAMP has the opposite effect in the intestine, and inhibits intestinal GC synthesis [26]. Furthermore, while SF-1 is the main transcriptional driver of adrenal GC synthesis, it is absent in the intestine, but functionally replaced by its close homolog liver receptor homolog 1 (LRH-1) [26-28]. Interestingly, in the intestine LRH-1 appears to contribute to the maintenance of the intestinal homeostasis via different mechanisms. Highly expressed in the stem cell region of the crypts, it not only regulates intestinal immune responses via local steroidogenesis, but contributes also to the renewal of the epithelial layer by controlling stemness, metabolism and proliferation of progenitors cells [29]. Most recently, it has been shown that LRH-1 also controls apoptosis resistance of intestinal epithelial cells (IEC) [30]. This is especially interesting as IECs are exquisitely sensitive to the apoptosis-inducing activity of TNF, and in the intestine LRH-1 will likely also counteract TNF-induced cell death, yet TNF also efficiently triggers LRH-1-regulated intestinal GC synthesis [31]. Thus, TNF critically contributes to the induction of intestinal GC synthesis initiated upon activation of $\mathrm{T}$ cells by anti- $\mathrm{T}$ cell receptor antibodies and virus, as well as macrophages by LPS (Fig. 2) [24, 27, 32]. In contrast to the role of TNF in triggering adrenal GC synthesis, this is likely a direct effect of TNF on the intestinal epithelium, as TNF triggers steroidogenesis in isolated intestinal crypts and immortalized IECs (Fig. 2) [25]. Of interest, both, TNF receptor 1 and 2 seem to be involved in TNF-mediated induction of intestinal GC synthesis [24]. While intestinal GC synthesis strongly depends on both, TNF and LRH-1, their mutual relationship and interaction is currently not understood (Fig. 2). While T cell activation- and colitis-induced intestinal GC synthesis depends on both, TNF and LRH-1, LPS-induced macrophage-mediated intestinal GC synthesis requires TNF, but not LRH-1 (Fig. 2) [24]. It is thus possibly that TNF and LRH-1 regulate two parallel and independent pathways, both enhancing intestinal GC synthesis.

\section{The role of intestinal glucocorticoids in the regulation of intestinal immune responses and inflammatory disorders}

The intestinal epithelial barrier is a major contact zone between luminal microbes and the intestinal immune system. Disruption of the intestinal epithelial barrier, especially in the colon, promotes invasion of the intestinal mucosa by 


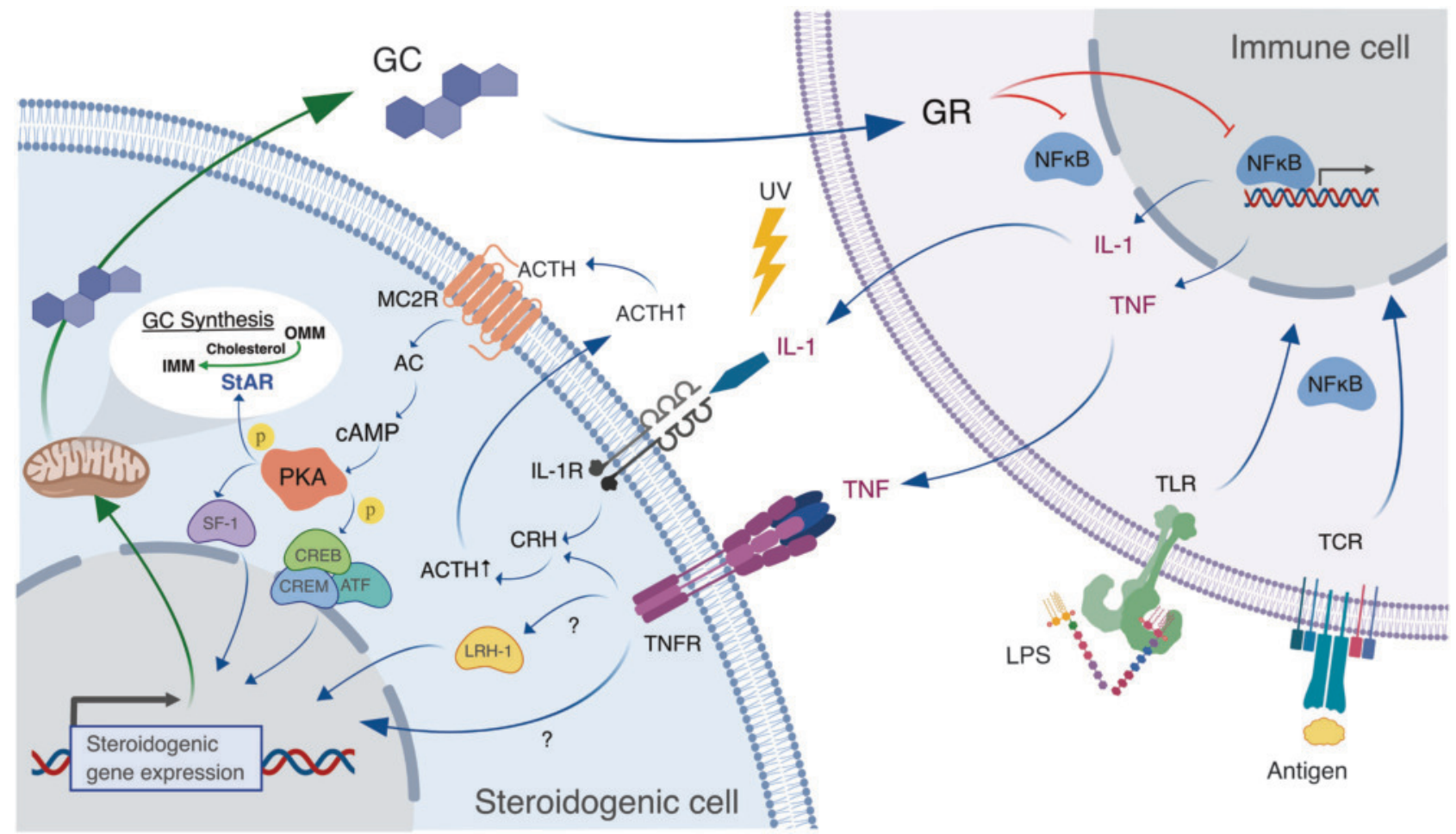

Fig. 2 Stimulation and regulation of extra-adrenal glucocorticoid synthesis. Antigens and bacterial components, e.g., lipopolysaccharides (LPS), activate immune cells via T cell receptor (TCR) or Tolllike receptors (TLR) signaling, respectively, and induce activation of the nuclear factor kappa-light-chain-enhancer of activated B cells $(\mathrm{NF \kappa B})$. This result in the induction and release of inflammatory cytokines, such as interleukin-1 (IL-1) or tumor necrosis factor (TNF). Extra-adrenal steroidogenic epithelial cells recognize the cytokines through specific receptors (TNFR and IL-1R). While in intestinal epithelial cells, TNFR signaling and the nuclear receptor liver receptor homolog 1 (LRH-1) are involved in steroidogenic enzyme expression, skin cells mainly translate inflammatory signaling into the release of corticotropin releasing hormone $(\mathrm{CRH})$, which induces the

microbes, and the activation of strategically distributed intestinal and infiltrating immune cells may result not only in host defense, but also in acute or even chronic intestinal inflammation. Inflammatory bowel disease (IBD), such as UC or Crohn's Diease (CD), represents a chronic intestinal disorder which requires a frequent treatment with antiinflammatory GC. Although still unclear, it is thought that environmental factors in combination with genetic predisposition may contribute to the pathogenesis of IBD. Recent research has shown that LRH-1 is involved in maintaining intestinal homeostasis through a variety of mechanisms. With the control of the intestinal GC synthesis through the regulation of steroidogenic enzyme expression, LRH-1 may represent a novel target in the therapeutic treatment of IBD, which may contribute to the "mucosal healing" (MH). Enhancing the activity of LRH-1 possibly gives new perspectives in boosting local GC synthesis, next to increased proliferation and stemness preservation of IEC to adrenocorticotropic hormone (ACTH). ACTH binds to the melanocortin 2 receptor (MC2R), initiates cAMP production via the adenylate cyclase (AC) and activates the cAMP-dependent protein kinase A (PKA), which ultimately directs the nuclear factor steroidogenic factor 1 (SF-1) or other nuclear factors (CREB and CREM ATF) to induce steroidogenesis via cAMP response elements (CRE) in the promoter of target genes. Activated PKA further phosphorylates StAR and enhances cholesterol transport from the outer mitochondrial membrane (OMM) to the inner mitochondrial membrane (IMM) to fuel the GC synthesis in the mitochondria. Synthesized GC are released and exert their anti-inflammatory actions via the GC receptor (GR) by inhibiting $\mathrm{NF \kappa B}$ and associated expression of pro-inflammatory cytokines

reestablish intestinal homeostasis. Considering the fact that not only LRH-1 but also the pro-inflammatory cytokine TNF drive intestinal GC synthesis, clinical therapies aiming at neutralizing TNF may result in adverse effects in the intestinal epithelium. In this regard, TNF was already shown to induce GC synthesis in acute models of experimental colitis [25]. However, further studies are required in order to carefully evaluate the role of TNF as a trigger of intestinal GC synthesis, especially in the context of chronic intestinal inflammation, such as UC and CD. It is also likely that inflammation-induced intestinal GC synthesis is finetuned by moderate amounts of pro-inflammatory cytokines, whereas overshooting levels of these cytokines may result in the suppression of intestinal GC synthesis promoting chronic inflammation. This possibly explains why the antiTNF therapy in IBD is very effective and promotes MH. However, although in many patients $\mathrm{MH}$ is achieved, up to $50 \%$ of patients develop clinical relapses within 1 year [33]. 
Evidence that TNF and T helper (Th) type 1 cytokines in general are key for the induction of intestinal GC has already been established, and future investigations will investigate the contribution of other pro-inflammatory cytokines in intestinal steroidogenesis. It tempting to speculate that by regulating local GC synthesis LRH-1 may not only limit local immune responses and inflammation, but may also drive longer lasting immune tolerance. It is wellknown that breakdown of immune tolerance is involved in the pathogenesis of IBD, and intestinal GC seem to be involved in maintaining intestinal barrier homeostasis via suppressing uncontrolled immune responses. In this regard, Bouquen et al. elegantly demonstrated that such a breakdown during UC was associated with impaired expression of the peroxisome proliferator-activated receptor- $\gamma$ (PPAR $\gamma)$. Their study revealed that IEC from patients with UC have decreased steroidogenic enzyme expression and associated cortisol production, and that LRH-1 controls PPAR $\gamma$ expression through GC synthesis in IEC [34]. This study further highlighted the role of LRH-1-controlled intestinal steroidogenesis in intestinal inflammation. Already earlier, Coste et al. provided evidence that LRH-1 is indeed an interesting therapeutic target in UC and CD, as LRH-1 expression in IEC seems to be essential for the homeostasis in the gastrointestinal tract [28, 30]. Overall, these studies demonstrated that intestinal de novo GC synthesis plays a crucial role in tissue homeostasis. Even though basal GC activity may be low, upon tissue activation through barrier disruption and immune cell activation IEC are able to increase local GC synthesis drastically.

\section{Lung}

The lung has a specialized anatomy for the exchange of gases, such as oxygen and carbon dioxide [35]. The tracheal and bronchial mucosa consists of a pseudostratified ciliated columnar epithelium, covered with a surface liquid for the clearance of inhaled particles [35]. In the bronchioles, type I epithelial cells are mostly responsible for gas exchange, whereas type II epithelial cells secrete surfactant, important for the surface tension of the bronchioles. In addition to its role in respiration, the pulmonary epithelium is known to have important functions in the activation of the innate and adaptive immune system [36]. The complex physical barrier provides the first line of defense against inhaled harmful substances or microbial pathogens [36]. Activation of epithelial cells ensures immediate alerting of immune cells and thus helps to maintain a functional barrier. However, prolonged or chronic activation of these cells can result in sustained airway inflammatory diseases, such as asthma [36]. While acute and chronic inflammation of the lung is frequently treated with GC, the lung is also a potent local source of endogenous GC [21]. Prior to the discovery of the lung as a steroidogenic tissue, the role of GC in lung development has been appreciated [37, 38]. GC stimulate the production of surfactants in type II epithelial cells, and thus regulate the stability of bronchioles [39]. Therefore, synthetic GC are used to accelerate lung development in preterm neonates immediately before birth [38]. Next to their role in lung development and differentiation, GC are also involved in the regulation of local immune responses in the mature lung. Already in 1999 first evidences for the expression of steroidogenic enzymes in the mouse lung were reported [40], and in the following years the expression of all steroidogenic enzymes necessary for the generation of corticosterone was experimentally verified [21, 41]. Importantly, in 2012 our group described for the first time that the lung produces large amounts of GC in response to immune cell activation, suggesting that locally produced GC may have a similar role in the regulation of pulmonary immune responses and inflammation, as in the intestine [21].

\section{Stimulation and regulation of glucocorticoid synthesis in lung epithelial cells}

The regulation of steroidogenic enzyme expression in the lung is so far rather unexplored. Hostettler et al. showed that in vivo immune cell activation by LPS or anti-CD3 antibody, or direct administration of TNF, resulted in increased production of corticosterone in ex vivo lung cultures [21]. Interestingly, however, while immune cell stimulation results in a strong transcriptional upregulation of steroidogenic enzymes in the gut, only Cyp11a1 was found to be induced in the lung. In contrast, most steroidogenic enzymes seem to be expressed at high basal levels and remain constant upon immune cell activation. A major exception is Cyp11b1, an enzyme required for de novo synthesis of GC form cholesterol, which was barely detectable. The high-expression levels of Hsd11b1 in comparison to Cyp11b1 suggested that reactivation of serum-derived dehydrocorticosterone to corticosterone is a more prominent pathway of local GC synthesis in the lung than de novo synthesis from cholesterol [21]. This hypothesis is in line with the observation that adrenalectomized mice were unable to produce GCs in the lung upon anti-CD3 antibody administration [21]. Furthermore, lung tissue was able to convert ${ }^{14} \mathrm{C}$-labeled dehydrocorticosterone to corticosterone, but not deoxycorticosterone to corticosterone. These findings indicate that the lung and the intestine are both capable of producing GC, yet via different enzymatic pathways. Interestingly, Provost and Tremblay (2005) investigated expression levels of steroidogenic enzymes in the lung during fetal development of mice. They 
found high expression levels of the enzymes required for de novo GC synthesis from cholesterol at gestation day (GD) 15. However, from GD 16 onwards, expression of these enzymes gradually decreased, whereas the expression of Hsd11b1 strongly increased. These observations may partially explain the results of Hostettler et al., since adult mouse lung seems to relay on corticosterone reactivation rather than de novo synthesis from cholesterol [21]. The reason why the lung employs a different strategy is currently not understood. Considering the effects of TNF administration on lung GC synthesis, it is likely that other pro-inflammatory cytokines, e.g., IL-1 $\beta$, regulate Hsd11b1 expression, and thereby promote local GC synthesis. Airway epithelial cells seem to sense their environment via the expression of Toll-like receptors [36]. Their activation by microbial products leads to the production of anti-microbial and anti-viral peptides, as well as pro-inflammatory cytokines and chemokines [36]. Especially, thymic stromal lymphopoetin, B cell-activating factor of the TNF family, and IL-33 (an IL-1 family member) were shown to shape a Th 2 type response and induce $\operatorname{IgA}$, $\operatorname{IgE}$, and $\operatorname{IgM}$ production in B cells [36]. Although the effect of these cytokines is mainly pro-inflammatory, it is feasible to believe that they may have a similar role in the induction of GC synthesis in the lung as TNF in the intestine. The relative absence of Cyp11b1 in the lung in comparison to the intestine suggests also a different transcriptional control of local GC synthesis. The lung expresses only low levels of LRH-1, which are further decreased upon immune cell activation [21]. Importantly, LRH-1 haploinsufficient mice, which show strongly reduced intestinal GC synthesis, reveal normal lung GC synthesis in response to anti-CD3 injection, indicating that LRH-1 has not a critical role in the transcriptional control of lung GC synthesis [21, 27]. Given that also SF-1 is expressed at only very low levels, it is very likely that lung GC synthesis is controlled by yet to be identified transcription factors [42].

\section{The role of local glucocorticoids in the regulation of immune responses and inflammatory diseases of the lung}

The role of GC in the control of lung development has been studied extensively over the last decades. The discovery of Liggins and Howie already in 1972 showing that prenatal administration of GC reduced the severity of respiratory distress syndrome in preterm infants, has been a hallmark in biomedical science $[43,44]$. In addition to their therapeutic use in neonatology, the anti-inflammatory actions of GC are also widely used in the treatment of chronic inflammatory lung diseases, such as chronic obstructive pulmonary disease (COPD) or asthma [45]. In asthma patients, GC treatment results in an immediate improvement of acute symptoms, such as gasp, sputum production, variable airflow limitation and airway hyperresponsiveness [46]. Allergic asthma is a Th2-driven disease, potentially caused by an interplay between genetic and environmental factors [46]. It has been shown that in asthma patients the epidermal barrier is disrupted resulting in increased exposure to allergens and infections [46, 47]. Moreover, in vitro studies in epithelial cells from asthmatic patients revealed increased expression of pro-inflammatory transcription factors, such as NFkB, AP-1, STAT1, and STAT6 [48]. Importantly, the successful application of GC in the treatment of this disease points out the potential immunoregulatory role endogenous GC may have in the lung. Since GC exert their anti-inflammatory actions, among others, via the inhibition of NFKB, AP-1, STAT1, and STAT6, it is likely that a defective local GC synthesis in the lung may contribute to the severity of the disease due to the lack of this potentially important negative feedback loop mechanism. Besides direct application of GC, 11 $\beta$-HSD1 may present an interesting therapeutic target in drug development since targeting the induction or activation of $11 \beta$-HSD1 could increase local GC reactivation in the lung, thereby limiting side effects of conventional systemic GC therapies [21].

Importantly, $30 \%$ of COPD patients treated with inhaled corticosteroids develop a resistance to the therapy [49]. Clinical studies and animal models revealed evidence for the involvement of neutrophils, alveolar macrophages, lymphocytes, and mast cells in resistance development [49]. Resistant patients display increased levels of IL-8, matrix metallopeptidase 9 (MMP-9), macrophage migration inhibitory factor, phosphoinositide 3-kinase $\delta$ and GR $\beta$, which potentially further contributes to sustained inflammation and tissue destruction [49]. Increased lung inflammation is paralleled by reduced expression and activity of the GR $\alpha$. Despite recent progress in overcoming resistance by the use of combinational application of antioxidants with corticosteroids, further research is inevitable, especially to find treatments to completely cure the disease [49]. The analysis of locally produced GC in COPD patients could contribute to the investigation of corticosteroid resistance and drug development. Possible defects of local GC synthesis in COPD patients could cause aggravation of symptoms, similar to inflammatory disorders in the skin. However, this remains to be examined.

\section{Skin}

The skin is one of the largest organs of the human body in terms of tissue weight. In contrast to the lung and the intestine it develops from the ectodermal germ layer. As a complex organ, the skin fulfills diverse and vital functions, and it is constantly confronted with environmental challenges. It thereby protects the inner organs from 
mechanical, physical, chemical, and biological insults. The skin is build up by different layers, the epidermis, dermis, and hypodermis. The epidermis mainly consists of keratinocytes forming the superficial stratified squamous layer, which provides resistance against mechanical forces, such as sloughing and abrasion, protects from damaging chemicals and radiation, and from pathogens entering the body. Additionally, the skin epithelial barrier prevents the body's water loss and regulates the temperature through several skin appendages, such as hair and sweat glands. It is not only involved in physical protection of the body, but also recognizes harmless environmental factors and signals through different sensation receptors converting them to neuroendocrine signals. Processing these external, but also internal, signals necessitates several versatile systems to suitably respond to harmful, respiratory harmless factors. In fact, many years of research revealed that the skin represents a local neuroendocrine system, capable of producing different kind of neuropeptides, signaling molecules, and hormones. Increasing evidence shows that different cellular components of the skin, most notably keratinocytes, express several cytochrome P450 enzymes and synthesize de novo $\mathrm{GC}$ in the similar way as the adrenal glands, but independently of them [50-54]. Keratinocytes were reported to metabolize cholesterol to pregnenolone, indicating that the skin is indeed able to produce steroids de novo. Of particular interest, the cellular skin components also locally produce $\mathrm{CRH}$ and POMC-derivatives, such as ACTH, which trigger their cognate receptors locally in the skin (Fig. 2) [55-57]. Thus, the skin is regarded as an equivalent of the systemic HPA axis, which enables the steroidogenic organ to locally respond in a neuroendocrine way to external and possibly also to internal stimuli. In fact, evidence was provided that the skin is able to cross-talk with the central HPA axis, suggesting the possibility that local skin and global steroidogenesis are able to regulate each other in a mutual way [58]. In this regard, it is still unclear to what extent both neuroendocrine systems affect each other, and further models are needed to reveal the potential of the combinatorial stress response system. These studies also raised the question regarding the role of skin-derived $\mathrm{GC}$ in the regulation of inflammatory skin disorders. The need for further in-depth investigations of this question was also stimulated by reports on deficiency of steroidogenic enzyme expression in skin biopsies from patients with psoriasis or atopic dermatitis [53, 54].

\section{Stimulation and regulation of glucocorticoid synthesis in the skin}

The characterization of skin GC synthesis and its control has been largely dominated by the dermatopathologist
Andrzej Slominski. He and his team showed in the past that the cutaneous GC synthesis lies under the hormonal control of a local skin-specific HPA axis, which, in fact, was shown to express all elements necessary to regulate local GC synthesis. Steroidogenic enzyme expression and GC synthesis in the skin seem to be tightly controlled by various factors, such as POMC-derived ACTH and CRH, or proinflammatory cytokines, such as IL-1 $\beta$ [59]. Steroidogenesis in the skin was shown to be directly linked to the action of $\mathrm{CRH}$, which stimulates ACTH production, resulting in the activation of a cAMP/PKA-dependent pathway (Fig. 2). However, recent publications demonstrated that also skin activation through ultraviolet (UV) radiation, mechanical injury or inflammatory cytokines, such as IL-1 $\beta$, can induce cutaneous de novo GC synthesis (Fig. 2) [52, 60]. Whether this is directly mediated through cytokine receptors on the GC-producing target cells as in the adrenal gland, or due to increased release of $\mathrm{CRH}$ and the following cAMP/PKA pathway signaling, is not fully understood yet. However, UV radiation was reported to induce $\mathrm{CRH}$ secretion in the skin, which results in the induction of GC synthesis indicating that $\mathrm{CRH}$ may initiate steroidogenesis. CRH release is also associated with several skin insulting signals, including mechanical, chemical, and immunological stress, as it promotes the production of pro-inflammatory inflammatory cytokines, such as IL-6, and anti-microbial peptides [57]. At the same time, $\mathrm{CRH}$ also triggers the activation of the cAMP/PKA pathway, which results in a negative feedback via increased de novo GC synthesis in the skin, counteracting thereby pro-inflammatory reactions and preserving the skin barrier homeostasis [57, 60]. Most notably, all enzymes involved in the de novo GC synthesis as well as all the elements of the HPA axis are expressed in keratinocytes, indicating that cutaneous GC synthesis and its regulation by the local skin HPA axis occurs also within the epidermis [52, 53, 60-62]. In contrast to the adrenal GC synthesis and the central HPA axis, the cutaneous HPA elements, the nervous system, and the local skin immune system are already located within the skin and in close proximity, and are connected through its local neuroendocrine network. In fact, this allows an adequate response upon stress signaling in the epithelial cells of the skin, the cutaneous nervous system and the diverse cell subsets of the skin immune system. As the release of signaling molecules, neuropeptides, and hormones, such as anti-inflammatory GC, and their target cells are all located within the same tissue or even the same skin compartment, this further enables regulatory mechanisms via autocrine, paracrine and intracrine actions, uncoupling thereby the cutaneous processes from the systemic ones. Although it was shown that the cAMP/PKA pathway is crucial for the de novo GC synthesis in the skin, the role of steroidogenic factors controlling GC synthesis is still far less characterized. So far, 
SF-1 was shown to be expressed in the skin, in specific skin compartments as well as skin appendages [63, 64]. The finding of SF-1 expression in the skin is consistent with the observation that cutaneous steroidogenesis is predominantly regulated by the cAMP/PKA signaling pathway (Fig. 2). Thus, it is likely that SF-1, possibly together with other nuclear factors, is orchestrating steroidogenesis in the skin. However, further experimental studies are required to unravel and characterize the steroidogenic regulators and to assess their relevance in cutaneous GC synthesis. Furthermore, Slominski et al. [60] already addressed relevant differences between the regulatory mechanisms of the adrenal and the cutaneous GC synthesis. In this regard, CYP11A1 expression and conversion rate of cholesterol to pregnenolone was found to be lower in the skin. In addition, cholesterol transport in the adrenal glands and gonads is regulated by StAR. In the placenta this rate-limiting process is operated by the metastatic lymph node 64 protein (MLN64) [60], whereas the skin was shown to express both StAR and MLN64 [17, 60, 61, 65]. As discussed recently, the skin is, similar to the placenta, also deficient for the acute response phase upon ACTH-induced cAMP/PKA signaling pathway, which increases StAR activity and rapid pregnenolone synthesis $[60,61]$. Although the induction of de novo steroid synthesis in the skin resembles the classical adrenal GC synthesis, cutaneous steroidogenesis seems to occur at a slower rate.

The reason why the skin is geared with different regulators may be explained by its diverse physiological endocrine functions, next to the de novo production of GC. In addition to the GC synthesis in the skin, the steroidogenic regulators and enzymes are also involved in the production of other hormone-like substances, for example sex steroids, such as androgens and estrogens, or a variety of secosteroids and derivatives $[60,66,67]$. The overall challenges of the transcriptional regulation of hormone production in the skin may thus be substantially different from that in other steroidogenic tissues, such as the adrenals and the gut.

\section{The role of local glucocorticoids in inflammatory skin diseases}

Several inflammatory skin diseases develop in response to unbalanced skin immune homeostasis. In this regard, skin barrier defects and uncontrolled immune responses are known to contribute to the pathogenesis of inflammatory skin diseases. To date, mild-inflammatory skin diseases are treated with emollients and other topical therapeutics for maintaining or reestablishing the skin barrier. However, cases with severe skin inflammation and chronic features, such as eczema and psoriasis, still require therapies using potent anti-inflammatory drugs, such as GC or biologicals.
This raises the question regarding the role of local cutaneous GC synthesis in these processes. Psoriasis, a chronic inflammatory skin disorder with features of uncontrolled skin inflammation involving dominant Th1 and 17 type immune responses, is regarded as an autoimmune disease, and especially in psoriasis patients it has been shown that therapy with steroid and biologicals against the Th1/17 cytokines is very efficient $[68,69]$. The detection of IL-12/ IL-23 in psoriatic lesions and the characterization of their role in skin inflammation resulted in the effective therapeutic attempt to simultaneously target both cytokines by neutralizing with anti-IL-12/IL-23p40 monoclonal antibodies [70, 71]. However, Kulig et al. [71] discovered a protective role of IL-12 in psoriatic lesions by restricting the invasion of $\mathrm{V} \gamma 6 \gamma \delta \mathrm{T} 17$ cells, and they thereby questioned the common assumption of the primarily pro-psoriatic role of IL-12. Next generation sequencing of murine keratinocyte RNA derived from naïve and Aldara-treated (promotes a psoriasis-like skin inflammation) wild type or IL-12 receptor deficient mice revealed the regulation of psoriasisrelated genes by IL-12 [71]. The expression of over 1000 genes involved in tissue structure, keratinocyte differentiation, basement membrane integrity, and immune regulation was altered between the groups, and interestingly, over 900 genes were differentially expressed when IL-12 was applied to TNF-activated keratinocytes [71]. The affected pathways could be involved in the regulation of the IL-23/type 17 immune response, which supports a rather anti-psoriatic role of IL-12 [71]. It is thus tempting to speculate that IL-12 secretion by resident dendritic cells may induce de novo GC production in neighboring keratinocytes. If IL-12 signaling enhances GC synthesis in keratinocytes, it could be a mechanism to limit local inflammation. Increased local GC concentrations may reverse the $\mathrm{T}$ cell differentiation from Th1 to Th2, and thereby restore the homeostatic balance. However, this hypothesis remains to be investigated experimentally. Of interest in this regard, it has been shown that the expression of steroidogenic enzymes involved in de novo GC synthesis, such as StAR and CYP11A1, is downregulated in psoriasis skin compared to healthy skin biopsies [53, 54]. This indicates that deficiency of de novo synthesized GC is likely involved in pathogenesis of inflammatory skin diseases. However, it is still unclear whether downregulation of the GC synthesis pathway in these inflammatory skin diseases is cause or consequence, i.e., might be a secondary effect in response to uncontrolled immune cell activation. Nonetheless, studies by Sevilla et al. [72] indicate that skin GC are targeting not only skinresident immune cells, but also the epithelial barrier itself [73, 74]. This was demonstrated by employing keratinocyte-specific GR deletion in the skin [72-74]. Deficiency in keratinocyte-specific GR signaling resulted in impaired skin development, keratinocyte differentiation and 
barrier organization [72-74]. Importantly, the epithelial GR signaling is also involved in the complex process of wound healing, and in addition to the GR, the MC receptor seems to contribute to the regulation of skin homeostasis $[72,75$, 76].

The cutaneous neuroendocrine system is not only capable of autonomously producing GC under the control of the local HPA-like axis, but is also able to cross-talk with the systemic HPA axis and thereby with the adrenal GC synthesis [58]. As already described by Slominski et al. [60], the skin and its local neuroendocrine system are able to react to UV radiation with increased activation of the local HPA axis and enhanced steroidogenesis. This observation suggests that skin GC synthesis, beside the local vitamin D synthesis, contributes to the therapeutic effects of clinical UV-based phototherapies, and may also explain the efficacy of phototherapies in immunosuppression and rebuilding of the epidermal barrier homeostasis [77-79]. This is especially efficient in the treatment of inflammatory skin disorders, such as psoriasis and sclerosis, as combinatorial therapies with GC, vitamin D analogs as well as retinoids are still the mainstay in topical therapy [80-84]. They share both anti-inflammatory and anti-proliferative effects, and are even enhancing/potentiating each other's action [85-87].

Thus, regulating skin homeostasis via local de novo synthesis of GC in keratinocytes may also have more systemic consequences on the status of the immune system. In particular, autoimmune diseases of the skin affect often also other organs, e.g., as seen in cutaneous lupus erythematosus skin sclerosis. Thus, reestablishing the endogenous GC synthesis in the skin not only opens a new therapeutic possibility in the treatment of inflammatory skin diseases, but provides also new insight into the role and mechanism of steroidogenesis in the skin in the regulation of local and systemic inflammation. Furthermore, it could contribute to our understanding of other physiological processes in the skin, such as wound healing, potentially stimulating also novel developments in the field of tissue-engineering and skin grafts.

\section{De novo GC synthesis vs. reactivation from derivatives}

The availability of bioactive GC via de novo synthesis or reactivation from decay metabolites appears to be differentially regulated in different extra-adrenal tissues. In contrast to their embryonic stage, the mature thymus and lung seem to rely more on $11 \beta$-HSD1-mediated GC reactivation rather than CYP11B1-mediated de novo synthesis, whereas the intestine and the skin appear to prefer de novo GC upon immune cell activation [21, 88]. Intestinal GC synthesis was shown to be regulated by LRH-1 and to be induced by proinflammatory cytokines, such as TNF. Resulting de novo GC synthesis has been associated with the control of intestinal inflammation, as shown in different experimental models of IBD. While de novo GC synthesis in the intestine has been investigated in detail, considerably less is known about the role of $11 \beta$-HSD1 in the GC synthesis in IEC, and its relevance for the pathogenesis of IBD $[89,90]$. Recently, a transcriptome analysis demonstrated that HSD11B1 expression was found to be generally increased in inflamed intestinal tissue of patients with $\mathrm{CD}$ compared to noninflamed normal mucosa and healthy controls [91]. Another study investigating the expression of $11 \beta$-HSD1 and $11 \beta$ HSD2 enzymes in patients with IBD revealed a trend towards higher 11 $\beta$-HSD1 expression levels in inflamed tissue compared to non-inflamed tissue [92]. Remarkably, this study also revealed a substantial decrease of $11 \beta$-HSD2 expression in IBD tissue compared to control tissue, suggesting that an increase in local GC levels may also be obtained by limiting the GC deactivation pathway [92]. Similar results were observed by Stegk et. al. [93] demonstrating pronounced upregulation of $11 \beta$-HSD1 in tissue samples from UC and CD patients, whereas $11 \beta$-HSD2 was found to be decreased. Thus, besides the important role of de novo GC synthesis in intestinal inflammation, the local regulation of $\mathrm{GC}$ reactivation and deactivation may also be relevant and possibly underestimated. This may particularly be of clinical relevance as the activity of steroid-based drugs may be modulated locally in the intestinal epithelium by inactivating and activating 11 -hydroxysteroid dehydrogenase enzymes.

Similar to the intestine, the skin is known to produce GC mainly via the de novo synthesis pathway. However, recent publications have documented that keratinocytes also express the $11 \beta-H S D 1 / 2$ enzyme system. Importantly, Terao et al. have shown that $11 \beta-H S D 1 / 2$ are involved in various physiological processes of the skin and specifically in keratinocytes, where these enzymes regulate cell proliferation, wound healing and skin inflammation induced by UV radiation and haptens [94-98]. Despite the cutaneous expression of $11 \beta$-HSD1, GC reactivation seem to play a minor role during immune cell activation and contact hypersensitivity (CHS), as shown by a low-dose hapteninduced CHS in a mouse model with keratinocyte-specific gene deletion of Hsd11bl [95]. However, the authors stated that at higher hapten doses no differences between wildtype and Hsd11b1-deficient mice were observed, suggesting that de novo GC synthesis might play a more essential role in inhibiting CHS reactions. It should also be considered that the enzymes involved in de novo GC synthesis vs. GC reactivation are differentially expressed by keratinocytes within the epidermis. Whereas $11 \beta$-HSD1 expression is predominantly found in supra-basal and more superficial 
layer of the epidermis, Cyp $11 \mathrm{~b} 1$ is predominantly expressed by basal layer keratinocytes $[52,98]$.

Although skin GC de novo synthesis and reactivation has been characterized to some detail, their respective roles in physiological and pathophysiological processes are still not fully understood. As described earlier, GC activation in the skin by $11 \beta-H S D 1$ seems to be involved in various cutaneous processes, however, its overall impact and relevance seem to be minor as it largely depends on GC metabolite supply from the circulation (e.g., cortisone and dehydrocorticosterone), whereas de novo GC synthesis seem to be constitutive, yet inducible, tissue-autonomous and may serve different functions in the skin by dictating proliferation and differentiation of keratinocytes, and barrier homeostasis, thereby affecting also the adjacent nervous and the skin immune system. Furthermore, it is still unclear and thus speculative how much systemic inactive GC metabolites are effectively reactivated in the epidermis, as most of 11 $\beta$-HSD1-expressing keratinocytes reside in the supra-basal or more superficial layer, and are progressing into differentiation stages. In contrast, keratinocyte-specific de novo GC synthesis in the basal layer allows autocrine and paracrine GC signaling, which also enables different GR-mediated actions depending on the local concentrations.

Taken together, different extra-adrenal organs seem to prefer different ways to provide their local environment with bioactive GC as they also clearly differ in their individual needs of GR-mediated actions. Although most of the extra-adrenal tissue are expressing enzymes for both de novo GC synthesis and reactivation of GC metabolites, their preferred mechanism of generating bioactive GC seems to depend on the local requirements and conditions.

\section{Clinical relevance and perspectives of extra- adrenal glucocorticoids}

A detailed understanding of molecular processes in the synthesis of endogenous GC is key to develop strategies how to target them in pathophysiological conditions, especially in inflammatory diseases at epithelial barriers. Lately, basic research not only investigated the synthesis of systemic GC, but also brought the extra-adrenal GC synthesis in focus. This is of importance as understanding the pathways of GC synthesis, and their enzymatic and transcriptional control may enable us to specifically fine-tune tissuespecific local GC synthesis, and thereby local immune responses and inflammation (Fig. 2). Based on the findings that $11 \beta$-HSD1 reactivates GC, inactive synthetic GC, such as prednisone, are now regularly used for the treatment of patients with inflammatory diseases. The conversion of inactive GC to their active metabolites occurs mostly in the liver, due to its high expression of $11 \beta-H S D 1$. Since the broad use of prednisone in clinical therapies of various inflammatory diseases, specific attempts in better controlling the release of bioactive GC have led to the so-called "modified-release" prednisone [99-101]. Considering the circadian rhythm and suppression of the HPA axis by negative feedback loops, these novel modifications aim to optimize the release of prednisone in order to minimize GC and $\mathrm{MC}$ receptor-mediated side effects and to develop a more time-controlled GC therapy [99-101]. The delayed release of these modified GC in the gut, e.g., prednisone and hydrocortisone, allows for example to ameliorate the typical morning pain and joint stiffness in patients with rheumatoid arthritis [99-101]. Interestingly, this may also help to improve clinical symptoms of IBD patients, considering the proposed role of $11 \beta-\mathrm{HSD} 1$ in the intestine of UC and CD patients. Thus, release-modified GC could stay longer in the intestine and may be more readily reactivated by IEC, restricting the activation of the GR to the local environment. Our steadily increasing knowledge of the mechanisms controlling intestinal GC synthesis and local reactivation may provide future opportunities in pharmacologically exploiting the local steroidogenesis in the gut, the lung, and the skin. Clinical therapies for inflammatory skin diseases are equipped with a broad availability of different synthetic and modified GC. Many of them are based on the structure of prednisolone with different modifications in order to facilitate the metabolism in deeper skin layers and to reduce thereby major GC side effects. Whereas $\mathrm{AD}$ patients are often treated with various GC with low to moderate potency, such as hydrocortisone, prednicarbate, clobetasone butyrate or mometasonfuroat, clinical therapies for patients with psoriasis include high-potency steroids, such as clobetasol propionate or betamethasone. The requirement of topical GC application seems to be quite different among the skin disorders as it also depends on the quality of the disease state and its clinical symptoms. This also shows that specific targeting/enhancing de novo GC synthesis or GC reactivation in the skin still requires functional analysis in order to implement novel drug inventions, for example the use of modified and epidermal-specific, inactive GC, similar to prednisone, to drive the activity of epidermal $11 \beta$-HSD1. On the other hand, reactivating/enhancing the enzymatic machinery of skin GC synthesis necessitates the activation of the cAMP/PKA pathway and its steroidogenic regulators, which leads to the proposal of several compounds, such as ACTH or forskolin. However, targeting these pathways specifically in keratinocytes is still challenging and needs to be elucidated in further investigations. While manipulating local GC synthesis may possibly not be as effective in suppressing inflammatory responses as high-dose systemic treatment with synthetic GC, it is very likely that also side effects of chronic GC treatment will be drastically reduced. 
Acknowledgments The authors greatly appreciate all scientific discussions with colleagues in the field of extra-adrenal glucocorticoid synthesis and nuclear receptors over the past 20 years, which contributed to this review. This work was supported by a research grant from the German Science Foundation (DFG, BR3369/9-1) to TB.

\section{Compliance with ethical standards}

Conflict of interest The authors declare that they have no conflict of interest.

\section{References}

1. McKay LI, Cidlowski JA. Cross-talk between nuclear factor-кB and the steroid hormone receptors: mechanisms of mutual antagonism. Mol Endocrinol. 1998. https://doi.org/10.1210/ mend.12.1.0044.

2. Ray A, Prefontaine KE. Physical association and functional antagonism between the p65 subunit of transcription factor NFkappa B and the glucocorticoid receptor. Proc Natl Acad Sci. 1994. https://doi.org/10.1073/pnas.91.2.752.

3. Yang-Yen HF, Chambard JC, Sun YL, Smeal T, Schmidt TJ, Drouin J, et al. Transcriptional interference between c-Jun and the glucocorticoid receptor: mutual inhibition of DNA binding due to direct protein-protein interaction. Cell. 1990;62:1205-15.

4. Diamond MI, Miner JN, Yoshinaga SK, Yamamoto KR. Transcription factor interactions: selectors of positive or negative regulation from a single DNA element. Science. 1990;249:126672.

5. Erlacher M, Michalak EM, Kelly PN, Labi V, Niederegger H, Coultas L, et al. BH3-only proteins Puma and Bim are ratelimiting for gamma-radiation- and glucocorticoid-induced apoptosis of lymphoid cells in vivo. Blood. 2005;106:4131-8.

6. Boldizsar F, Talaber G, Szabo M, Bartis D, Palinkas L, Nemeth $\mathrm{P}$, et al. Emerging pathways of non-genomic glucocorticoid (GC) signalling in T cells. Immunobiology. 2010;215:521-6.

7. Turnbull AV, Rivier CL. Regulation of the hypothalamicpituitary-adrenal axis by cytokines: actions and mechanisms of action. Physiol Rev. 1999;79:1-71.

8. Honma S. The mammalian circadian system: a hierarchical multi-oscillator structure for generating circadian rhythm. J Physiol Sci. 2018;68:207-19.

9. Schibler U, Gotic I, Saini C, Gos P, Curie T, Emmenegger Y, et al. Clock-talk: interactions between central and peripheral circadian oscillators in mammals. Cold Spring Harb Symp Quant Biol. 2016;80:223-32.

10. Kalsbeek A, van der Spek R, Lei J, Endert E, Buijs RM, Fliers E. Circadian rhythms in the hypothalamo-pituitary-adrenal (HPA) axis. Mol Cell Endocrinol. 2012;349:20-29.

11. Pon LA, Hartigan JA, Orme-Johnson NR. Acute ACTH regulation of adrenal corticosteroid biosynthesis. Rapid accumulation of a phosphoprotein. J Biol Chem. 1986;261:13309-16.

12. Clark BJ, Soo SC, Caron KM, Ikeda Y, Parker KL, Stocco DM. Hormonal and developmental regulation of the steroidogenic acute regulatory protein. Mol Endocrinol. 1995;9:1346-55.

13. Arakane F, King SR, Du Y, Kallen CB, Walsh LP, Stocco DM, et al. Phosphorylation of steroidogenic acute regulatory protein (StAR) modulates its steroidogenic activity. J Biol Chem. 1997;272:32656-62.
14. Ruggiero C, Lalli E. Impact of ACTH signaling on transcriptional regulation of steroidogenic genes. Front Endocrinol. 2016;7:24.

15. Parker KL, Rice Da, Lala DS, Ikeda Y, Luo X, Wong M, et al. Steroidogenic factor 1: an essential mediator of endocrine development. Recent Prog Horm Res. 2002;57:19-36.

16. Parker KL. The roles of steroidogenic factor 1 in endocrine development and function. Mol Cell Endocrinol. 1998;145:1520.

17. Parker KL, Ikeda Y, Luo X. The roles of steroidogenic factor-1 in reproductive function. Steroids. 1996;61:161-5.

18. Wang XL, Bassett M, Zhang Y, Su Y, Clyne C, White PC, et al. Transcriptional regulation of human 11ß-hydroxylase (hCYP11B1). Endocrinology. 2000;1:3587-94.

19. Rice DA, Aitken LD, Vandenbark GR, Mouw AR, Franklin A, Schimmer BP, et al. A cAMP-responsive element regulates expression of the mouse steroid 11 $\beta$-hydroxylase gene. J Biol Chem. 1989;264:14011-5.

20. Talabér G, Jondal M, Okret S. Extra-adrenal glucocorticoid synthesis: immune regulation and aspects on local organ homeostasis. Mol Cell Endocrinol. 2013;380:89-98.

21. Hostettler N, Bianchi P, Gennari-Moser C, Kassahn D, Schoonjans K, Corazza N, et al. Local glucocorticoid production in the mouse lung is induced by immune cell stimulation. Allergy. 2012;67:227-34.

22. Mittelstadt PR, Taves MD, Ashwell JD. Cutting edge: de novo glucocorticoid synthesis by thymic epithelial cells regulates antigen-specific thymocyte selection. J Immunol. 2018;200:1988-94

23. Cima I, Corazza N, Dick B, Fuhrer A, Herren S, Jakob S, et al. Intestinal epithelial cells synthesize glucocorticoids and regulate T cell activation. J Exp Med. 2004;200:1635-46.

24. Noti M, Corazza N, Tuffin G, Schoonjans K, Brunner T. Lipopolysaccharide induces intestinal glucocorticoid synthesis in a TNFalpha-dependent manner. FASEB J. 2010;24:1340-6.

25. Noti M, Corazza N, Mueller C, Berger B, Brunner T. TNF suppresses acute intestinal inflammation by inducing local glucocorticoid synthesis. J Exp Med. 2010;207:1057-66.

26. Mueller M, Atanasov A, Cima I, Corazza N, Schoonjans K, Brunner T. Differential regulation of glucocorticoid synthesis in murine intestinal epithelial versus adrenocortical cell lines. Endocrinology. 2007;148:1445-53.

27. Mueller M, Cima I, Noti M, Fuhrer A, Jakob S, Dubuquoy L, et al. The nuclear receptor LRH-1 critically regulates extraadrenal glucocorticoid synthesis in the intestine. J Exp Med. 2006;203:2057-62.

28. Coste A, Dubuquoy L, Barnouin R, Annicotte J-S, Magnier B, Notti M, et al. LRH-1-mediated glucocorticoid synthesis in enterocytes protects against inflammatory bowel disease. Proc Natl Acad Sci. 2007;104:13098-103.

29. Botrugno OA, Fayard E, Annicotte JS, Haby C, Brennan T, Wendling $\mathrm{O}$, et al. Synergy between LRH-1 and $\beta$-catenin Induces G1cyclin-mediated cell proliferation. Mol Cell. 2004:15:499-509.

30. Bayrer JR, Wang H, Nattiv R, Suzawa M, Escusa HS, Fletterick RJ, et al. LRH-1 mitigates intestinal inflammatory disease by maintaining epithelial homeostasis and cell survival. Nat Commun. 2018;9:4055.

31. Delgado ME, Grabinger T, Brunner T. Cell death at the intestinal epithelial front line. FEBS J. 2016;384:2701-19.

32. Huang J, Jia R, Brunner T. Local synthesis of immunosuppressive glucocorticoids in the intestinal epithelium regulates antiviral immune responses. Cell Immunol. 2018;334:1-10.

33. Cintolo M. Mucosal healing in inflammatory bowel disease: maintain or de-escalate therapy. World J Gastrointest Pathophysiol. 2016;7:1-16. 
34. Dubuquoy L, A Jansson E, Deeb S, Rakotobe S, Karoui M, Colombel JF, et al. Impaired expression of peroxisome proliferator-activated receptor yin ulcerative colitis. Gastroenterology. 2003;124:1265-76.

35. Tomashefski JF, Farver CF. Anatomy and histology of the lung. In: Tomashefski JF Jr., Cagle PT, Farver CF FA (arg). Dail and Hammar's Pulmonary Pathology. New York, NY: Springer; 2008. p. 20-48.

36. Kato A, Schleimer RP. Beyond inflammation: airway epithelial cells are at the interface of innate and adaptive immunity. Curr Opin Immunol. 2007;19:711-20.

37. Taylor KM, Ray DW, Sommer P. Glucocorticoid receptors in lung cancer: new perspectives. J Endocrinol. 2016;229:R17R28.

38. Bolt RJ, Weissenbruch MM, Van, Lafeber HN. Glucocorticoids and lung development in the fetus and preterm infant. Pediatr Pulmonol. 2001;91:76-91.

39. Nkadi PO, Merritt TA, Pillers DAM. An overview of pulmonary surfactant in the neonate: genetics, metabolism, and the role of surfactant in health and disease. Mol Genet Metab. 2009;97:95101.

40. Pazirandeh A, Xue Y, Rafter I, Sjövall J, Jondal MOS. Paracrine glucocorticoid activity produced by mouse thymic epithelial cells. FASEB J. 1999;13:893-901.

41. Provost PR, Tremblay Y. Genes involved in the glucocorticoid synthesis are transiently expressed in the developing lung. Endocrinology. 2005;146:2239-45.

42. Nishimura M, Naito S, Yokoi T. Tissuespecific mRNA expression profiles of human nuclear receptor subfamilies. Drug Metab Pharm. 2004;19:135-49.

43. Bird AD, Mcdougall ARA, Seow B, Hooper SB, Cole TJ. Glucocorticoid regulation of lung development: lessons learned from conditional GR knockout mice. Mol Endocrinol. 2015;29:158-71.

44. Liggins GC, Howie RN. A controlled trial of antepartum glucocorticoid treatment for prevention of the respiratory distress syndrome in premature infants. Pediatrics. 1972;50:515-25.

45. Chalmers JD, Poole C, Webster S, Tebboth A, Dickinson S, Gayle A. Assessing the healthcare resource use associated with inappropriate prescribing of inhaled corticosteroids for people with chronic obstructive pulmonary disease (COPD) in GOLD groups A or B: an observational study using the Clinical Practice Research Data. Respir Res. 2018;19:1-9.

46. Heijink IH, Nawijn MC, Hackett TL. Airway epithelial barrier function regulates the pathogenesis of allergic asthma. Clin Exp Allergy. 2014;44:620-30.

47. Gon Y, Hashimoto S. Role of airway epithelial barrier dysfunction in pathogenesis of asthma. Allergol Int. 2018;67:12-17.

48. Hackett TL, Singhera GK, Shaheen F, Hayden P, Jackson GR, Hegele RG, et al. Intrinsic phenotypic differences of asthmatic epithelium and its inflammatory responses to respiratory syncytial virus and air pollution. Am J Respir Cell Mol Biol. 2011:45:1090-1100.

49. Jiang Z, Zhu L. Update on molecular mechanisms of corticosteroid resistance in chronic obstructive pulmonary disease. Pulm Pharmacol Ther. 2016;37:1-8.

50. Slominski A, Baker J, Ermak G, Chakraborty A, Pawelek J. Ultraviolet B stimulates production of corticotropin releasing factor (CRF) by human melanocytes. FEBS Lett. 1996;399:175-6.

51. Slominski a, Gomez-Sanchez CE, Foecking MF, Wortsman J. Active steroidogenesis in the normal rat skin. Biochim Biophys Acta. 2000;1474:1-4.

52. Vukelic S, Stojadinovic O, Pastar I, Rabach M, Krzyzanowska A, Lebrun E, et al. Cortisol synthesis in epidermis is induced by IL-1 and tissue injury. J Biol Chem. 2011;286:10265-75.
53. Hannen RF, Michael AE, Jaulim A, Bhogal R, Burrin JM, Philpott MP. Steroid synthesis by primary human keratinocytes; implications for skin disease. Biochem Biophys Res Commun. 2011;404:62-7.

54. Hannen R, Udeh-Momoh C, Upton J, Wright M, Michael A, Gulati A, et al. Dysfunctional skin-derived glucocorticoid synthesis is a pathogenic mechanism of psoriasis. J Invest Dermatol. 2017;137:1630-7.

55. Ermak G, Slominski A. Production of POMC, CRH-R1, MC1, and $\mathrm{MC} 2$ receptor mRNA and expression of tyrosinase gene in relation to hair cycle and dexamethasone treatment in the C57BL/6 mouse skin. J Invest Dermatol. 1997;108:160-5.

56. Slominski A, Ermak G, Mazurkiewicz JE, Baker JWJ. Characterization of corticotropin-releasing hormone $(\mathrm{CRH})$ in human skin. J Clin Endocrinol Metab. 1998;83:1020-4.

57. Slominski AT, Zmijewski MA, Zbytek B, Tobin DJ, Theoharides TC, Rivier J. Key role of CRF in the skin stress response system. Endocr Rev. 2013;34:827-84.

58. Skobowiat C, Slominski AT. UVB activates hypothalamicpituitary-adrenal axis in C57BL/6 mice. J Invest Dermatol. 2015;135:1638-48.

59. Slominski A, Wortsman J, Luger T, Paus R, Solomon S. Corticotropin releasing hormone and proopiomelanocortin involvement in the cutaneous response to stress. Physiol Rev. 2000;80:979-1020.

60. Slominski AT, Manna PR, Tuckey RC. On the role of skin in the regulation of local and systemic steroidogenic activities. Steroids. 2015;103:72-88.

61. Slominski A, Zbytek B, Nikolakis G, Manna PR, Skobowiat C, Zmijewski M, et al. Steroidogenesis in the skin: implications for local immune functions. J Steroid Biochem Mol Biol. 2013;137:107-23.

62. Cirillo N, Prime SS. Keratinocytes synthesize and activate cortisol. J Cell Biochem. 2011;112:1499-505.

63. Thiboutot D, Jabara S, McAllister JM, Sivarajah A, Gilliland K, Cong $\mathrm{Z}$, et al. Human skin is a steroidogenic tissue: steroidogenic enzymes and cofactors are expressed in epidermis, normal sebocytes, and an immortalized sebocyte cell line (SEB-1). J Invest Dermatol. 2003;120:905-14.

64. Patel MV, McKay IA, Burrin JM. Transcriptional regulators of steroidogenesis, DAX-1 and SF-1, are expressed in human skin. J Invest Dermatol. 2001;117:1559-65.

65. Slominski A, Zjawiony J, Wortsman J, Semak I, Stewart J, Pisarchik A, et al. A novel pathway for sequential transformation of 7-dehydrocholesterol and expression of the P450scc system in mammalian skin. Eur J Biochem. 2004;271:4178-88.

66. Zmijewski MA, Slominski AT. Neuroendocrinology of the skin: an overview and selective analysis. Dermatoendocrinol. 2011;3:3-10.

67. Slominski AT, Kim T-K, Li W, Yi A-K, Postlethwaite A, Tuckey RC. The role of CYP11A1 in the production of vitamin $\mathrm{D}$ metabolites and their role in the regulation of epidermal functions. J Steroid Biochem Mol Biol. 2014;144:28-39.

68. Elder JT. Genome-wide association scan yields new insights into the immunopathogenesis of psoriasis. Genes Immun. 2009;10:201-9.

69. Liu Y, Krueger JG, Bowcock AM. Psoriasis: genetic associations and immune system changes. Genes Immun. 2007;8:1-12.

70. Garcia VE, Chang M, Brandon R, Li Y, Matsunami N, CallisDuffin KP, et al. Detailed genetic characterization of the interleukin-23 receptor in psoriasis. Genes Immun. 2008;9:54655 .

71. Kulig P, Musiol S, Freiberger SN, Schreiner B, Gyülveszi G, Russo G, et al. IL-12 protects from psoriasiform skin inflammation. Nat Commun. 2016;7:13466. 
72. Sevilla LM, Pérez P. Roles of the glucocorticoid and mineralocorticoid receptors in skin pathophysiology. Int J Mol Sci. 2018;19:1906.

73. Whirledge S, DeFranco DB. Glucocorticoid signaling in health and disease: Insights from tissue-specific GR knockout mice. Endocrinology. 2018;159:46-61.

74. Sevilla LM, Latorre V, Sanchis A, Pérez P. Epidermal inactivation of the glucocorticoid receptor triggers skin barrier defects and cutaneous inflammation. J Invest Dermatol. 2013;133:361-70.

75. Boix J, Carceller E, Sevilla LM, Marcos-Garcés V, Pérez P. The mineralocorticoid receptor plays a transient role in mouse skin development. Exp Dermatol. 2016;25:69-71.

76. Bigas J, Sevilla LM, Carceller E, Boix J, Pérez P. Epidermal glucocorticoid and mineralocorticoid receptors act cooperatively to regulate epidermal development and counteract skin inflammation article. Cell Death Dis. 2018;9:588.

77. Krutmann J, Morita A. Mechanisms of ultraviolet (UV) B and UVA phototherapy. J Investig Dermatol Symp Proc. 1999;4:70-2.

78. Walker D, Jacobe H. Phototherapy in the age of biologics. Semin Cutan Med Surg. 2011;30:190-8.

79. Pavel S. Light therapy (with UVA-1) for SLE patients: is it a good or bad idea? Rheumatology. 2006;45:653-5.

80. Uva L, Miguel D, Pinheiro C, Antunes J, Cruz D, Ferreira J, et al. Mechanisms of action of topical corticosteroids in psoriasis. Int J Endocrinol. 2012;2012:561018.

81. Papp KA, Guenther L, Boyden B, Grønhøj Larsen F, Harvima RJ, Guilhou JJ, et al. Early onset of action and efficacy of a combination of calcipotriene and betamethasone dipropionate in the treatment of psoriasis. J Am Acad Dermatol. 2003;48:48-54.

82. Devaux S, Castela A, Archier E, Gallini A, Joly P, Misery L, et al. Topical vitamin D analogues alone or in association with topical steroids for psoriasis: a systematic review. J Eur Acad Dermatol Venereol. 2012;26:52-60.

83. Fleming C, Ganslandt C, Guenther L, Johannesson A, Buckley C, Simon JC, et al. Calcipotriol plus betamethasone dipropionate gel compared with its active components in the same vehicle and the vehicle alone in the treatment of psoriasis vulgaris: a randomised, parallel group, double-blind, exploratory study. Eur J Dermatol. 2010;20:465-71.

84. Menter A, Korman NJ, Elmets CA, Feldman SR, Gelfand JM, Gordon $\mathrm{KB}$, et al. Guidelines of care for the management of psoriasis and psoriatic arthritis. J Am Acad Dermatol. 2009;61:451-85.

85. Zhang Y, Leung DYM, Goleva E. Vitamin D enhances glucocorticoid action in human monocytes: Involvement of granulocyte-macrophage colony-stimulating factor and mediator complex subunit 14. J Biol Chem. 2013;288:14544-53.

86. Hidalgo AA, Trump DL, Johnson CS. Glucocorticoid regulation of the vitamin D receptor. J Steroid Biochem Mol Biol. 2010;121:372-5.

87. Cutolo M, Paolino S, Sulli A, Smith V, Pizzorni C, Seriolo B. Vitamin D, steroid hormones, and autoimmunity. Ann N Y Acad Sci. 2014;1317:39-46.
88. Taves MD, Hamden JE, Soma KK. Local glucocorticoid production in lymphoid organs of mice and birds: Functions in lymphocyte development. Horm Behav. 2017;88:4-14.

89. Kostadinova FI, Hostettler N, Bianchi P, Brunner T. Extraadrenal glucocorticoid synthesis in mucosal tissues and its implication in mucosal immune homeostasis and tumor development. In: Qian X (Ed.). In Glucocorticoids-New Recognition of Our Familiar Friend; 2012.

90. Kostadinova F, Schwaderer J, Sebeo V, Brunner T. Why does the gut synthesize glucocorticoids? Ann Med. 2014;46:490-7.

91. Hong SN, Joung JG, Bae JS, Lee CS, Koo JS, Park SJ, et al. RNA-seq reveals transcriptomic differences in inflamed and noninflamed intestinal mucosa of Crohn's disease patients compared with normal mucosa of healthy controls. Inflamm Bowel Dis. 2017;23:1098-108.

92. Hussey M, Holleran G, Smith S, Sherlock M, McNamara D. The role and regulation of the 11 beta-hydroxysteroid dehydrogenase enzyme system in patients with inflammatory bowel disease. Dig Dis Sci. 2017;62:3385-90.

93. Stegk JP, Ebert B, Martin HJ, Maser E. Expression profiles of human $11 \beta$-hydroxysteroid dehydrogenases type 1 and type 2 in inflammatory bowel diseases. Mol Cell Endocrinol. 2009;301:104-8.

94. Terao M, Katayama I. Local cortisol/corticosterone activation in skin physiology and pathology. J Dermatol Sci. 2016;84:11-16.

95. Terao M, Itoi S, Matsumura S, Yang L, Murota H, Katayama I. Local glucocorticoid activation by 11 b-hydroxysteroid dehydrogenase 1 in keratinocytes the role in hapten-induced dermatitis. Am J Pathol. 2016;186:1499-510.

96. Itoi-Ochi S, Terao M, Murota H, Katayama I. Local corticosterone activation by $11 \beta$-hydroxysteroid dehydrogenase 1 in keratinocytes: the role in narrow-band UVB-induced dermatitis. Dermatoendocrinol. 2016;8:1-12.

97. Murota H, Itoi S, Terao M, Matsui S, Kawai H, Satou Y, et al. Topical cholesterol treatment ameliorates hapten-evoked cutaneous hypersensitivity by sustaining expression of 11b-HSD1 in epidermis. Exp Dermatol. 2014;23:68-70.

98. Terao M, Murota H, Kimura A, Kato A, Ishikawa A, Igawa K, et al. 11b-Hydroxysteroid dehydrogenase-1 Is a novel regulator of skin homeostasis and a candidate target for promoting tissue repair. PLoS ONE. 2011;6:1-11.

99. Clarke L, Kirwan J. Efficacy, safety and mechanism of action of modified-release prednisone in rheumatoid arthritis. Ther Adv Musculoskelet Dis. 2012;4:159-66.

100. Buttgereit F. Novel glucocorticoids and glucocorticoid receptor ligands: teaching old drugs new tricks. Ann Rheum Dis. 2014;73:41.

101. Ursini F, Naty S, Bruno C, Grembiale RD. Old but good: modified-release prednisone in rheumatoid arthritis. Rev Recent Clin Trials. 2017;12:124-8. 